\title{
PRELIMINARY STUDY ON THE OCCURRENCE OF PTEN AND PIK3CA GENE MUTATIONS IN ENDOMETRIAL CANCER AMONG MALAYSIAN WOMEN
}

\author{
Subramaniam $\mathrm{KS}^{1,3}$, Wong $\mathrm{MS}^{1,3}$, Woo $\mathrm{YL}^{2,3}$, Mat Adenan $\mathrm{NA}^{2}$, Mohamed $\mathrm{Z}^{1}$, Chung $\mathrm{I}^{1,3}$ \\ Departments of ${ }^{1}$ Pharmacology and ${ }^{2}$ Obstetrics \& Gynecology, Faculty of Medicine, University of Malaya, Malaysia \\ ${ }^{3}$ University of Malaya Cancer Research Institute, University of Malaya, Malaysia
}

\author{
Correspondence: \\ Ivy Chung \\ Associate Professor, Department of Pharmacology, \\ Faculty of Medicine, University of Malaya, 50603 Kuala Lumpur, Malaysia. \\ Phone: 60-3-7967-6685. Fax: 60-3-7967-4791. \\ E-mail: ivychung@ummc.edu.my
}

\begin{abstract}
Genetic mutations in endometrial cancer (EC) have been extensively studied in the Western population but not much in Asian cohorts. This study has demonstrated that PTEN and PIK3CA mutations are commonly found in EC among Malaysian women. Following RNA extraction from 20 cancerous and 18 non-cancerous tissues, the presence of mutations in 9 exons of PTEN and 3 exons of PIK3CA genes were detected using realtime PCR, accompanied by High Resolution Melt (HRM) analysis. Sequencing confirmed specificity of each PCR product. The mutations for both genes were detected in the samples with varying frequencies. Notably, all samples expressed mutation of PTEN at exon 7 but none in exon 4. Further analysis demonstrated that strong concurrent mutations occurred between exons 7 of PTEN with exon 20 region 1 of PIK3CA gene (90\%). Our data showed mutations are present in EC and not the non-cancerous tissues. Larger samples are being collected to validate this observation.
\end{abstract}

Keywords: Uterine cancer, Malaysian, genetic abnormalities

\section{Introduction}

Worldwide, endometrial cancer (EC) ranks sixth among commonly diagnosed female cancer with 288,000 new cases and mortality rate from 1.7 to $2.4 \%$ per 100,000 women in 2008 (1). It is also the top gynecological malignancy in the United States, making it the 8th leading cause of cancer death among women worldwide. American Cancer Society has estimated about 49,560 new cases of EC and 8,190 women succumbing to this disease in 2013 (2). In Malaysia, National Cancer Incidence reported that EC contributed to $4.1 \%$ of total cancer cases involving women in 2007 . This was a rise from $3.3 \%$ in 2003 . There are 2 types of EC namely Type 1 and Type 2, with the former having better prognosis and survival rate of $83 \%$ compared to $25 \%$ for the latter (3). Several factors are thought to be linked to the rising trend of EC incidence worldwide, including the increase in obesity incidence, unopposed exposure to estrogen due to hormonal treatment after menopause and nullparity (4).

In addition to the environmental and hormonal factors, genetics may represent an important key regulator in EC cancer occurrence and progression. Oncogenes and tumor suppressor genes are the two gene classifications in which their mutations affect the development of cancer cells (5). Activation of oncogenic genes such as catalytic subunit $\alpha$ of phosphatidylinositol-4,5-bisphosphate 3-kinase (PIK3CA) gene, and inactivation of tumor suppressor genes such as Phosphatase and tensin homolog (PTEN) gene, are thought to be the key genetic changes involved in endometrial cancer development $(6,7)$.

PTEN is responsible for controlling cell growth by regulating the cell cycle at $G_{1} / S$ checkpoint, and loss of PTEN gene function was reported in $83 \%$ of EC cases (8). PTEN often acts with phosphatidylinositol-4,5-bisphosphate 3-kinase (PI3K) gene to control the activity of AKT signaling, which is important in proliferating cancer cells. Up to $26 \%$ of tumors harboring PTEN mutation also have mutation in PIK3CA. Mutation in PIK3CA can lead to an additive effect on PI3K signaling activation. In fact, it was found that the mutation of PIK3CA were more common in tumor with PTEN mutations compared to those without (7). Additionally, contribution of PIK3CA and PTEN gene mutations are often implicated 
with endometrial cancer, both individually as well as by co-existing together (7,9-14). Given the importance of these genes in EC progression, we aim to investigate in this preliminary study whether such mutations can be detected in a small cohort of patients with endometrial cancer admitted to University Malaya Medical Center (UMMC).

\section{Methodology}

\section{Ethics statement}

The study was approved by the Ethical Committee of University Malaya Medical Center (Ref No. 865.19). Written informed consent was obtained from all participants.

\section{Tissue inclusion and exclusion criteria}

All the cancer tissues used in this study were from patients with confirmed endometrial cancer, while the control (non-tumor) tissues were from patients with non-tumor conditions such as post-menopausal bleeding, and from dilation and curettage samples. All the cancer tissues were confirmed by pathologist to be type 1 endometrial cancer of endometriod adenocarcinoma. Patients who were pregnant, under-age and had been diagnosed for other types of cancer were excluded from this study.

\section{Human endometrium tissue processing and RNA extraction}

All 38 snap frozen tissue samples (20 cancer and 18 controls) were collected from the University of Malaya, Faculty of Medicine, Biobank Unit. These tissues were cut to $2 \mathrm{~mm}$ length and transferred to $1.5 \mathrm{ml}$ microcentrifuge tubes containing $100 \mu \mathrm{l}$ phosphate buffered saline (Life Technologies, NY, USA). Next, equivalent amount of stainless steel beads with diameter of $1.6 \mathrm{~mm}$ (Next Advance, New York, USA) were added into the tubes. The tissues were then homogenized by using a bullet blender (Next Advance, New York, USA). Total RNA were extracted from homogenized tissues using TRIsure (Bioline, London, UK) according to the manufacture's protocol, and the yield of the RNA was quantified using NanoDrop ND-2000 (Thermo Fisher Scientific Inc, Massachusetts, USA). Total RNA was converted into cDNA using RevertAid First Strand cDNA Synthesis Kit (Thermo Fisher Scientific Inc, Massachusetts, USA).

\section{Real Time PCR and analysis of High Resolution Melt (HRM)}

Real Time PCR was performed using $A B I$ StepOne Plus (Applied Biosystem, California, USA) in 40 cycles. Each PCR reaction included5x HOT FIREPol EvaGreen qPCR Mix (Solis Biodyne, Tartu, Estonia), $10 \mathrm{pmol} / \mu \mathrm{l}$ forward and reverse primers, $10 \mathrm{ng} / \mu \mathrm{l} \mathrm{cDNA}$ template and PCR grade $\mathrm{H}_{2} \mathrm{O}$ prior to HRM analysis using High Resolution Melt Software v3.0.1
(Applied Biosystem, California, USA). Exons 1-9 of PTEN gene and exons 9 and 20 of PIK3CA gene were analyzed for mutation. All PCR reactions were performed in duplicates, and data shown were from at least 2 independent experiments. Primers used are shown in Table 1.

Table 1: List of primer sequences

\begin{tabular}{|c|c|c|}
\hline Exon & Forward $\left(3^{\prime} \rightarrow 5^{\prime}\right)$ & Reverse $\left(3^{\prime} \rightarrow 5^{\prime}\right)$ \\
\hline \multicolumn{3}{|l|}{ PTEN } \\
\hline 1 & CAGAAGAAGCCCCGCCACCAG & AGAGGAGCAGCCGCAGAAATG \\
\hline 2 & TTTCAGATATTTCTTTCCTTA & AACAAGAATATAAAACATCAA \\
\hline 3 & TAATTTCAAATGTTAGCTCAT & AAGATATTTCAAGCATACAA \\
\hline 4 & GTTTGTTAGTATTAGTACTTT & ACAACATAGTACAGTACATC \\
\hline $5(1)$ & ACCTGTTAAGTTTGTATGCAAC & CTTTCCAGCTTTACAGTGAA \\
\hline $5(2)$ & GCTAAGTGAAGATGACAATCA & TCCAGGAAGAGGAAAGGAAA \\
\hline 6 & CATAGCAATTTAGTGAAATAACT & GATATGGTTAAGAAAACTGTTC \\
\hline 7 & TGACAGTTTGACAGTTAAAGG & GGATATTTCTCCCAATGAAAG \\
\hline $8(1)$ & TTAAATATGTCATTTCATTTCTTTT & TTGCTTTGTCAAGATCATT \\
\hline $8(2)$ & GTGCAGATAATGACAAGGAATA & TCATGTTACTGCTACGTAAAC \\
\hline 9 & TTCATTTTAAATTTTCTTTCT & TTTTCATGGTGTTTTATCCCTC \\
\hline \multicolumn{3}{|c|}{ PIK3CA } \\
\hline 9 & GATTGGTTCTTTCCTGTCTCTG & CCACAAATATCAATTTACAACCATTG \\
\hline $20(1)$ & TGGGGTAAAGGGAATCAAAAG & CCTATGCAATCGGTCTTTGC \\
\hline $20(2)$ & TTGCATACATTCGAAAGACC & GGGGATTTTTTGTTTTGTTTTTG \\
\hline
\end{tabular}

\section{Sequencing of PCR Products}

PCR products were purified using MSB Spin PCRapace kit (Stratec, Berlin, Germany) and the validity of each product is confirmed with sequencing analysis (AIT Biotech Pte Ltd, Singapore).

\section{Results}

\section{Patient demographic distribution}

Cancer tissues used in this study were all from type 1 endometrial cancer with varying stages and histogrades. Stage $1 \mathrm{~A}$ and Grade 2 were the predominant classifications, with 8 and 10 cases, respectively (Table 2a). Control tissues were collected from non-tumor conditions: postmenapausal bleeding (10 cases), endometrial hyperplasia (3 cases) and endometrial fibroid (5 cases) (Table $2 b$ ). The patient cohort in this study comprised of 3 ethnic groups (Malays, Chinese and Indians) and their age ranged from 30 to 79 years old. As shown in Table 3, the Malays represented the majority (50\%) of the cancer patients compared to $22.2 \%$ in the control group. Approximately $30 \%$ and $20 \%$ of the remaining cancer cases were Chinese and Indian, compared to about $56 \%$ and $22 \%$ in control cases. Stratification analysis according to age showed that almost $80 \%$ of cancer patients were above 50 years old, consistent with the aetiology of this disease that mostly affecting post-menopausal women (Table 4). 
Table 2(a): Staging and grading information for cancer tissues in this study

\begin{tabular}{|l|l|c|c|c|c|}
\hline \multirow{2}{*}{ CANCER TISSUES } & \multicolumn{5}{|c|}{ Histogrades } \\
\cline { 3 - 7 } & & 1 & 2 & 3 & Total \\
\hline \multirow{4}{*}{ Tumor stages } & $1 \mathrm{~A}$ & 3 & 5 & 0 & $\mathbf{8}$ \\
\cline { 2 - 6 } & $1 \mathrm{~B}$ & 0 & 3 & 1 & $\mathbf{4}$ \\
\cline { 2 - 6 } & 2 & 1 & 2 & 0 & $\mathbf{3}$ \\
\cline { 2 - 6 } & $3 \mathrm{C} 1$ & 1 & 0 & 0 & $\mathbf{1}$ \\
\cline { 2 - 6 } & $4 \mathrm{~A}$ & 1 & 0 & 1 & $\mathbf{2}$ \\
\cline { 2 - 6 } & $4 \mathrm{~B}$ & 0 & 0 & 2 & $\mathbf{2}$ \\
\cline { 2 - 6 } & Total & $\mathbf{6}$ & $\mathbf{1 0}$ & $\mathbf{4}$ & $\mathbf{2 0}$ \\
\hline
\end{tabular}

Table 2(b): Control tissues classifications for tissues used in this study

\begin{tabular}{|l|c|}
\hline CONTROL TISSUES & \\
\hline Conditions & No. \\
\hline Post-menapausal bleeding & 10 \\
\hline Hyperplasia & 3 \\
\hline Fibroid & 5 \\
\hline Total & $\mathbf{1 8}$ \\
\hline
\end{tabular}

Table 3: $\quad$ Patients demography according to race distribution among cases and controls

\begin{tabular}{|l|c|c|c|c|}
\hline \multirow{2}{*}{} & \multicolumn{3}{|c|}{ Race } & \multirow{2}{*}{ Total } \\
\cline { 2 - 4 } & Malay & Chinese & Indian & \\
\hline Cases & $10(50)$ & $6(30)$ & $4(20)$ & $\mathbf{2 0}$ \\
\hline Control & $4(22.2)$ & $10(55.6)$ & $4(22.2)$ & 18 \\
\hline Total & $\mathbf{1 4}$ & $\mathbf{1 6}$ & $\mathbf{8}$ & $\mathbf{3 8}$ \\
\hline
\end{tabular}

*Data are given as frequency (percentage)

Table 4: Patients demography according to age distribution among cases and controls

\begin{tabular}{|l|c|c|c|c|c|c|}
\hline \multirow{2}{*}{} & \multicolumn{5}{|c|}{ Age group } & \multirow{2}{*}{ Total } \\
\cline { 2 - 7 } & $\mathbf{3 0 - 3 9}$ & $\mathbf{4 0 - 4 9}$ & $\mathbf{5 0 - 5 9}$ & $\mathbf{6 0 - 6 9}$ & $\mathbf{7 0 - 7 9}$ & \\
\hline Cases & $3(15)$ & $1(5)$ & $6(30)$ & $5(25)$ & $5(25)$ & $\mathbf{2 0}$ \\
\hline Control & $5(27.7)$ & $7(38.9)$ & $5(27.8)$ & $1(5.6)$ & $0(0)$ & $\mathbf{1 8}$ \\
\hline Total & $\mathbf{8}$ & $\mathbf{8}$ & $\mathbf{1 1}$ & $\mathbf{6}$ & $\mathbf{5}$ & $\mathbf{3 8}$ \\
\hline
\end{tabular}

*Data are given as frequency (percentage)

\section{Occurrence of tumor mutation}

We screened for presence of mutations in the tissues as summarized in Table 5(a) and (b). Our findings were verified by capillary sequencing. Analysis of PIK3CA gene mutations showed highest occurrence in exon 20 region 1 (90\%) followed by exon 9 (65\%) and lastly in exon 20 region 2
(55\%). Exons of PTEN gene exhibited different percentages of mutation occurrences with all samples being mutated in exon $7(100 \%)$ compared to no samples being mutated in exon $4(0 \%)$. None of these mutations were detected in the control tissues ( $0 \%)$.

Table 5(a): Frequency of mutations occurrence in cases and controls of PIK3CA gene

\begin{tabular}{|l|c|c|}
\hline \multirow{2}{*}{ PIK3CA } & $\begin{array}{c}\text { Cases } \\
(\mathrm{n}=20)\end{array}$ & $\begin{array}{c}\text { Controls } \\
(\mathrm{n}=18)\end{array}$ \\
\cline { 2 - 2 } & $\mathbf{n}(\%)$ & $\mathbf{n}(\%)$ \\
\hline PIK3CA-9 & $13(65)$ & \multirow{2}{*}{$0(0)$} \\
\hline PIK3CA 20-1 & $18(90)$ & \\
\hline PIK3CA 20-2 & $11(55)$ & \\
\hline
\end{tabular}

*Data are given as frequency (percentage). RNA from cases and controls were extracted and subjected to real time PCR followed by HRM analysis. Frequency above represents total number of patients with mutation.

Table 5(b): Frequency of mutations occurrence in cases and controls of PTEN gene

\begin{tabular}{|c|c|c|}
\hline \multirow{2}{*}{ PTEN } & Cases $(n=20)$ & Controls $(n=18)$ \\
\hline & n (\%) & n (\%) \\
\hline PTEN 1 & $15(75)$ & \multirow{11}{*}{$0(0)$} \\
\hline PTEN 2 & $2(10)$ & \\
\hline PTEN 3 & $2(10)$ & \\
\hline PTEN 4 & $0(0)$ & \\
\hline PTEN 5-1 & $16(80)$ & \\
\hline PTEN 5-2 & $14(70)$ & \\
\hline PTEN 6 & $13(65)$ & \\
\hline PTEN 7 & $20(100)$ & \\
\hline PTEN 8-1 & $5(25)$ & \\
\hline PTEN 8-2 & $18(90)$ & \\
\hline PTEN 9 & $6(30)$ & \\
\hline
\end{tabular}

*Data are given as frequency (percentage). RNA from cases and controls were extracted and subjected to real time PCR followed by HRM analysis. Frequency above represents total number of patients with mutation.

\section{Simultaneous PIK3CA and PTEN gene mutations}

We further analyzed the trends of simultaneous mutations occurrence between exons of a gene as well as between genes (Table 6). For PTEN, the highest concurrent mutation was between exon 7 and exon 8 region 2 (18/20 cases), while for PIK3CA, frequent concurrent mutation were found between exon 9 and exon 20 region 1 (11/ 20 cases). Simultaneous mutations analysis was also performed between the two genes. Exon 7 of PTEN gene showed strong co-occurrence with exon 20 region 1 of PIK3CA gene $(18 / 20$ cases $)$. 
Table 6: $\quad$ Simultaneous occurrence of mutation between exons and genes

\begin{tabular}{|c|c|c|c|c|c|c|c|c|c|c|c|c|c|c|}
\hline & \multicolumn{10}{|c|}{ PTEN } & \multicolumn{3}{|c|}{ PIKЗCA } \\
\hline & & 1 & 2 & 3 & 5 (1) & $5(2)$ & 6 & 7 & $8(1)$ & $8(2)$ & 9 & 9 & 20 (1) & $20(2)$ \\
\hline \multirow{10}{*}{ PTEN } & 1 & & 2 & 2 & 12 & 11 & 9 & 15 & 5 & 13 & 6 & 10 & 14 & 6 \\
\hline & 2 & & & 1 & 2 & 2 & 2 & 2 & 2 & 2 & 2 & 1 & 2 & 1 \\
\hline & 3 & & & & 2 & 1 & 2 & 2 & 2 & 2 & 2 & 1 & 2 & 2 \\
\hline & $5(1)$ & & & & & 12 & 10 & 16 & 5 & 14 & 5 & 9 & 15 & 7 \\
\hline & $5(2)$ & & & & & & 9 & 14 & 3 & 12 & 4 & 8 & 13 & 7 \\
\hline & 6 & & & & & & & 13 & 5 & 13 & 4 & 11 & 12 & 9 \\
\hline & 7 & & & & & & & & 6 & 18 & 6 & 13 & 18 & 11 \\
\hline & $8(1)$ & & & & & & & & & 5 & 3 & 4 & 5 & 2 \\
\hline & $8(2)$ & & & & & & & & & & 5 & 13 & 16 & 11 \\
\hline & 9 & & & & & & & & & & & 4 & 5 & 4 \\
\hline \multirow{3}{*}{ РІКЗСА } & 9 & & & & & & & & & & & & 11 & 9 \\
\hline & $20(1)$ & & & & & & & & & & & & & 9 \\
\hline & $20(2)$ & & & & & & & & & & & & & \\
\hline
\end{tabular}

*Data shown are frequency of concurrent occurrence; Bolded numbers represent more than $50 \%$ mutation co-occurrence

\section{Discussion}

In this study, we screened for various mutations of PTEN and PIK3CA genes in 20 endometrial cancer and 18 noncancerous endometrial tissues collected from Malaysian women. In addition, we also analyzed the patterns of mutation co-occurrence between exons in these genes. While contribution of these genes in endometrial cancer has been studied in depth in western population (15), such information are not available for Malaysian women. Such information may provide a significant clinical implication, as status of mutations in tumors may predict resistance of tumor cells against selected drug therapy.

PIK3CA gene mutation was highly expressed in our study, where it demonstrated at least $55 \%$ frequency in all three different exons examined. PIK3CA is an important catalytic subunit of the phosphatidylinositol 3-kinases (PI3Ks) that regulates cell proliferation, adhesion and survival (10). Many investigative agents, such as rapamycin, RAD001 and evorolimus that primarily blocks mTOR (a downstream molecule of PI3K pathway) are also known to inhibit the action of PIK3CA $(16,17)$. Determining the status of mutation in genes is crucial before making any clinical decision, considering the recent report that suggested mutation in another oncogenic gene, the K-ras gene may be the cause of cancer cells developing resistance towards mTOR inhibitors (16). This emphasizes the importance of mutation screening prior to decisions of therapeutic interventions. Interestingly, it was demonstrated that mutations in PIK3CA gene can lead to constant activation of PI3K pathway, and therefore blocking the effect of $\mathrm{MTOR}$ inhibitors $(12,17)$. It was also shown recently, mutation in exon 20 of this gene is associated with high-grade endometrial cancer and that another mutation site in this gene, H1047R correlated with shorter survival (18).
Our analysis further demonstrated that this gene (PIK3CA exon 2 region 1 ) occurred in the presence of PTEN gene mutations (exon 7), consistent with previous findings $(7,12)$. It is worthwhile to note that co-existence of these two gene mutations seems to be frequent in endometrial cancer but is quite rare in other cancers (13). It was also reported that PTEN mutations are observed specifically in endometrial cancer but not in other gynecological malignancies (14) and that it is frequently found in type 1 endometrial cancer (18). In contrast to cancer types, where PTEN mutation translates to increased metastatic potential, such mutation in endometrial cancer may be associated with a favorable survival (9).

Endometrial cancer is in part a genetic-driven process, and many clinical decisions are now being made based on the genetic profiling of the tumor. While the sample size in this study is relatively small, our data strongly suggests that mutations in PTEN and PIK3CA genes can be detected in the endometrial cancer samples presented by Malaysian women but not in controls, and this may imply that the etiology of this tumor in this region share similarities with those from the Western countries. A more comprehensive subsequent analysis is warranted to validate this finding in a larger sample cohort and to investigate the association of these mutations with the prognosis of these women. Determination of these mutations may allow for a more informed clinical diagnosis and to make a choice of the therapy required.

\section{Conclusion}

This preliminary study showed the presence of mutations in PTEN and PIK3CA among patients with endometrial cancer admitted to UMMC, with strong co-occurrence of 
mutations between exon 7 of PTEN with exon 20 region 1 of PIK3CA gene.

\section{Acknowledgements}

This study was supported by University Malaya Research Grant (UMRG 336/11HTM) and HIR-UMCRI/MOHE/ MED-12 and HIR-MOHE-E00025-20001. Tissue collection was undertaken by the University of Malaya, Faculty of Medicine, Biobank Unit.

\section{References}

1. Ferlay J, Shin H, Bray F, Forman D, Mathers Ca, Parkin D. GLOBOCAN 2008 v2.0, Cancer Incidence and Mortality Worldwide: IARC CancerBase No. 10 2010. 2010; http://globocan.iarc.fr. Accessed 29 September, 2012.

2. Society AC. Cancer Facts \& Figures 2013. American Cancer Society. 2012.

3. Zainal Ariffin O, Nor Saleha Ibrahim T. National Cancer Registry Report Malaysia Cancer Statistics-Data and Figures. National Cancer Registry, Ministry of Health, Malaysia: Ministry of Health Malaysia;2007.

4. Kaaks R, Lukanova A, Kurzer MS. Obesity, Endogenous Hormones, and Endometrial Cancer Risk A Synthetic Review. Cancer Epidemiol Biomarkers Prev. 2002;11:1531.

5. Chow AY. Cell Cycle Control by Oncogenes and Tumor Suppressors: Driving the Transformation of Normal Cells into Cancerous Cells. Nature Education. 2010;3(9):7.

6. Bokhman JV. Two pathogenetic types of endometrial carcinoma. Gynecol Oncol. . 1983;15:10-17.

7. Oda K, Stokoe D, Taketani Y, McCormick F. High frequency of coexistent mutations of PIK3CA and PTEN genes in endometrial carcinoma. Cancer Res. 2005;65:10669-10673.

8. Bansal N, Yendluri V, Wenham R. The molecular biology of endometrial cancers and the implications for pathogenesis, classification, and targeted therapies. Cancer Control. 2009;16(1):8-13.
9. Risinger JI, Hayes K, Maxwell GL, et al. PTEN mutation in endometrial cancers is associated with favorable clinical and pathologic characteristics. Clin. Cancer Res. 1998;4:3000-3010.

10. Catasus L, Gallardo A, Cuatrecasas M, Prat J. PIK3CA mutations in the kinase domain (exon 20) of uterine endometrial adenocarcinomas are associated with adverse prognostic parameters. Modern Pathology. 2008;21:131-139.

11. Dong $\mathrm{Y}$, Yang $\mathrm{X}$, Wong $\mathrm{O}$, et al. PIK3CA mutations in endometrial carcinomas in Chinese women: phosphatidylinositol 3'-kinase pathway alterations might be associated with favorable prognosis. Hum Pathol. 2012;43(8):1197-1205.

12. Hayes MP, Wang H, Espinal-Witter R, et al. PIK3CA and PTENMutations in Uterine Endometrioid Carcinoma and Complex Atypical Hyperplasia. Clin Cancer Res. 2006;12(20):5932-5935.

13. Sun $\mathrm{H}$, Enomoto T, Fujita M, et al. Mutational Analysis of the PTEN Gene in Endometrial Carcinoma and Hyperplasia. Am J Clin Pathol. 2001;115:32-38.

14. Tashiro H, Blazes M, Wu R, Cho K, Bose S, Wang $\mathrm{S}$. Mutations in PTEN are frequent in endometrial carcinoma but rare in other common gynecological malignancies. . Cancer Res. 1997;57:3935-3940.

15. Krakstad C, Birkeland E, Seidel D, et al. HighThroughput Mutation Profiling of Primary and Metastatic Endometrial Cancers Identifies KRAS, FGFR2 and PIK3CA to Be Frequently Mutated. PLoS ONE. 2012 7(12):e52795.

16. Mohseni M, Park BH. PIK3CA and KRAS mutations predict for response to everolimus therapy: now that's RAD001. The Journal of Clinical Investigation. 2010;120(8):2656-2658.

17. Ray-Coquard I, L Favier BW, Roemer-Becuwe C, et al. Everolimus as second- or third-line treatment of advanced endometrial cancer: ENDORAD, a phase II trial of GINECO. British Journal of Cancer 108. 2013;108:1771-1777.

18. Garcia-Dios DA, Lambrechts D, Coenegrachts L, et al. High-throughput interrogation of PIK3CA, PTEN, KRAS, FBXW7 and TP53 mutations in primary endometrial carcinoma. Gynecol Oncol. 2013;128:327-334. 